\title{
Erworbener Nystagmus mit Scheinbewegungen - was tun?
}

\section{A. Kirsch, A. Neugebauer, J. Fricke und W. Rüssmann}

Zentrum für Augenheilkunde der Universität Köln

Schielbehandlung und Neuroophthalmologie

Erworbener Nystagmus, der zumeist durch Hirnstammdefekte entsteht, erzeugt erhebliche visuelle Irritationen, die durch drei verschiedene Therapiekonzepte gemildert werden können: pharmakologisch, optisch oder durch Augenmuskelchirurgie. Pharmakologisch werden bei einzelnen Nystagmustypen in Zusammenarbeit mit Neurologen zentral wirksame Medikamente eingesetzt, in besonderen Fällen kann auch Botulinum-Toxin zur Anwendung kommen. Als optische Hilfen dienen besonders Einstärkengläser für Ferne und Nähe, die den Betroffenen den Durchblick in jeder gewünschten Blickrichtung ermöglichen. In einigen Fällen mit begleitenden Binokularstörungen sind Prismen sinnvoll. Bei Patienten mit Nystagmusverstärkung im Abblick helfen Lese- und Stehpulte bei Naharbeiten. Operativ kommen bei Vorhandensein von peripher gelegenen Nystagmusruhezonen blickverlagernde Eingriffe im Sinne von Kestenbaum oder Anderson in Frage. Fehlen dem Patienten eigene Kompensationsmechanismen, kann die Nystagmusamplitude durch großstreckige Rücklagerungen oder Fadenoperationen reduziert werden.

\section{Zentral-vestibuläre Nystagmusformen: Ätiologie, Diagnostik und topodiagnostische Bedeutung \\ U. Pichler}

Zusammenfassung. Zentral-vestibuläre Nystagmen entstehen durch eine Tonusimbalanz des vestibuloocularen Reflexes in der horizontalen, vertikalen oder torisonalen Ebene. Sie entstehen meist bei unterschiedlichen Hirnstamm- oder Kleinhirnläsionen, wobei eine genaue topodiagnostische Zuordnung ohne Berücksichtigung weiterer okulomotorischer Symptome oft nicht genau möglich ist.

$\mathrm{Zu}$ den zentral-vestibulären Nystagmusformen gehören: Down-Beat-Nystagmus, Up-Beat-Nystagmus, See-Saw-Nystagmus und Hemi-See-Saw-Nystagmus, periodisch alter- nierender Nystagmus, torsioneller Nystagmus und zentraler Lagenystagmus.

Es wird im Vortrag jeweils auf die einzelnen Nystagmusformen eingegangen, ihre charakteristischen Merkmale, sowie die zugrunde liegende Pathogenese werden aufgezeigt. Ebenso wird bei jeder Nystagmusform auf die Ätiologie hingewiesen und mögliche Begleitsymptome erwähnt. Kurzvideos von einzelnen zentral-vestibulären Nystagmusformen dienen der besseren Visualisierung.

\section{Wenn der Hirnstamm schwindelt - Zur Lokalisation von Hirnstammerkrankungen C. Frenzel \\ Orthoptik, Neurologische Klinik, Ludwig-Maximilians-Universität München, Klinikum Großhadern (Leiter der Klinik: Prof. Dr. Th. Brandt)}

Zusammenfassung. Funktionsstörungen zentral-vestibulärer Strukturen und deren Verschaltungen sind Ursache zentralvestibulärer Nystagmusformen sowie statischer Augenfehlstellungen (z. B. Ocular-tilt-reaction). Diese vestibulären klinischen Zeichen gehen meist einher mit subjektiv empfundenem Schwindelgefühl des Patienten.

Entsprechend der räumlichen Anordnung der Bogengänge im Innenohr sowie der zentralen Verschaltung des vestibulookulären Reflexes (VOR) in 3 verschiedenen Raumebenen können vestibuläre Symptome klinisch eingeteilt werden: Störungen des VOR in der Horizontalebene (Yaw) führen zu horizontalem Nystagmus und horizontaler Blickdeviation, und Störungen des VOR in der Rollebene führen zu rotie- rendem Nystagmus und Ocular-tilt-reaction (OTR). Hierbei gilt, dass Störungen in der Yaw- und Rollebene durch einseitige Hirnstammläsionen, Störungen in der Pitchebene, immer durch beidseitige Hirnstammläsionen bedingt sind.

Topographisch können Defekte in der Rollebene im gesamten Hirnstamm auftreten, Defekte in der Pitchebene nur an bestimmten Orten im oberen und unteren Hirnstamm und Defekte in der Yawebene nur im unteren Hirnstamm in einem relativ kleinen Bereich. In Kombination mit einer Augenmuskelparese oder anderen zentralen Okulomotorikstörungen wie BRN oder Blickfolgesakkadierungen können zentral vestibuläre Symptome helfen die Lage einer Läsion zu bestimmen. 